\title{
Pairing fluctuations in trapped Fermi gases
}

\author{
Luciano Viverit, ${ }^{1,2}$ Georg M. Bruun, ${ }^{3}$ Anna Minguzzi, ${ }^{4}$ and Rosario Fazio ${ }^{4}$ \\ ${ }^{1}$ Dipartimento di Fisica, Università di Milano, Via Celoria 16, 20133, Milan, Italy \\ ${ }^{2}$ CRS-BEC INFM and Dipartimento di Fisica, Università di Trento, I-38050 Povo, Italy \\ ${ }^{3}$ Niels Bohr Institute, Blegdamsvej 17, 2100 Copenhagen, Denmark \\ ${ }^{4}$ NEST-INFM \& Scuola Normale Superiore, Piazza dei Cavalieri 7, I-56126 Pisa, Italy
}

\begin{abstract}
Fluctuations of the amplitude of the order parameter govern the properties of superconducting systems close to the critical transition temperature. In the BCS regime we examine the contribution of these pairing fluctuations to the superfluid order parameter for harmonically confined atomic Fermi gases. In the limit of small systems we obtain an expression for the space dependence of the fluctuations, in good agreement with the results of numerical calculations. In this limit we also predict a parity effect, i.e. that pairing fluctuations should show a maximum or a minimum at the centre of the trap, depending on the value of the last occupied shell being even or odd. We finally propose to detect pairing fluctuations by measuring the density-density correlation function as evaluated after a ballistic expansion of the gas.
\end{abstract}

PACS numbers:

Progress in trapping and cooling of gases of fermionic atoms [1] have paved the way to explore a rich variety of phenomena. A major experimental effort nowadays is directed towards the observation of the superfluid transition. The presence of a Feshbach resonance allows to study the behaviour of Fermi gases both in the weakly and in the strongly interacting regime by varying an externally applied magnetic field. Away from the resonance the effective interatomic interaction is well represented by a contact pseudopotential $g \delta(\mathbf{r})$, with $g=4 \pi \hbar^{2} a / m$ and $a$ being the $s$-wave scattering length. A number of papers have explored the properties of a Fermi system across the resonance with particular attention to the BCS-BEC crossover and the strongly-interacting limit $k_{F} a \rightarrow \pm \infty$ [2], with $k_{F}=(3 \pi n)^{1 / 3}$ and $n$ being the atomic density. In the regime $k_{F} a \rightarrow 0^{+}$bosonic molecules have been already observed [3] and they have been reported to undergo condensation [4]; current experiments start to explore the $a<0$ region [5]. It has been suggested that by adiabatically tuning the scattering length from positive to negative it could be possible to reach a BCS-like regime [6]. Here we concentrate on such weak-coupling regime $k_{F} a \rightarrow 0^{-}$[].

Mean-field BCS theory predicts the appearence of a non-vanishing order parameter $\Delta_{\mathrm{BCS}}(T)$ below a critical temperature $T_{c}$ which depends strongly both on the interaction strength and on the geometry of the system [8]. The scale of variation of the order parameter is the coherence length $\xi=\hbar^{2} k_{F} / m \pi \Delta_{\mathrm{BCS}}(0) \gg n^{-1 / 3}, m$ being the atomic mass, to be compared with the size $R$ of the cloud under confinement (which we suppose harmonic). In the case $\xi \ll R$ of very large traps mean-field theory is very accurate in describing the physical properties of the condensate. The experiments with atomic gases, however, are performed on systems of adjustable size ranging from clouds of about $10^{4}-10^{7}$ atoms in harmonic traps to droplets of few tens of atoms in each lattice site of an op- tical lattice. One expects that by lowering the number of atoms in the trap (or equivalently when $\xi \geq R$ ) fluctuations of the order parameter can become important close to the critical temperature. This is the regime we discuss in this work. Above $T_{c}$ fluctuations signal the onset of the Cooper pair instability which will eventually drive the system to the superfluid state. In superconductors the role of fluctuations both on the thermodynamics and on the transport properties has been studied in great details both theoretically and experimentally and we refer to Ref. [9] for a comprehensive review of the field.

The aim of this Letter is to formulate the theory of pairing fluctuations in the case of trapped Fermi gases. This problem resembles that studied in zero-dimensional superconductors (i.e. of radius smaller that the coherence length) [10]. There are however important differences that we are going to highlight. First of all pairing fluctuations are inhomogeneous due to the presence of the trap; we will show below that they are enhanced in the center of the trap. Another important issue is how to detect fluctuations. In conventional superconductors they dominate the behaviour of most thermodynamical and transport properties (e.g. paraconductivity, density of states, specific heat, ...) close to $T_{c}[9]$. In trapped atoms, however, those quantities are very hard or impossible to measure. As a clear signature for the detection of pairing fluctuations we propose to analyze density-density correlations which were recently suggested by Altman et al. 11] as a probe of many-body states in ultra-cold atoms.

We assume that the system is composed of fermions in two internal states $\sigma=\uparrow, \downarrow$ with equal numbers and attractive intercomponent interactions, which we shall describe by the contact pseudopotential $g \delta(\mathbf{r})$. Correspondingly, we shall consider only pairing fluctuations in $s$-wave. This is the main collisional channel at ultralow temperatures, while $s$-wave interactions among atoms belonging to the same internal state are forbidden by the 
Pauli principle. The second-quantized Hamiltonian reads

$$
\begin{aligned}
\hat{H} & =\sum_{\sigma=\uparrow, \downarrow} \int d^{3} r \psi_{\sigma}^{\dagger}(\mathbf{r})\left(-\frac{\hbar^{2}}{2 m} \nabla^{2}+V(\mathbf{r})-\mu\right) \psi_{\sigma}(\mathbf{r}) \\
& +g \int d^{3} r \psi_{\uparrow}^{\dagger}(\mathbf{r}) \psi_{\downarrow}^{\dagger}(\mathbf{r}) \psi_{\downarrow}(\mathbf{r}) \psi_{\uparrow}(\mathbf{r}),
\end{aligned}
$$

where $\psi_{\sigma}$ are the fermionic field operators, $\mu$ is the chemical potential, $V(\mathbf{r})$ the confining potential and $g<0$. Close to $T_{c}$ the most important contribution beyond mean field comes from pair fluctuations, we therefore treat the density correlations at the Hartree level and add the Hartree fields $W_{\sigma}(\mathbf{r})=g n_{-\sigma}(\mathbf{r})$ to the singleparticle part of the Hamiltonian.

By introducing the auxiliary field $\Delta$ through a Hubbard-Stratonovich transformation 12 the fermion fields can be integrated over. Close to the critical temperature the partition function becomes $Z=$ $Z_{0} \int d\left[\Delta^{*}\right] d[\Delta] e^{-S\left[\Delta^{*}, \Delta\right] / \hbar}$, where $Z_{0}$ is the partition function of the gas in the Hartree approximation and the effective action to quartic order is

$$
\begin{aligned}
S & =-\hbar \beta \int d \mathbf{r}_{1} d \mathbf{r}_{2} \Delta^{*}\left(\mathbf{r}_{1}\right) A\left(\mathbf{r}_{1}, \mathbf{r}_{2}\right) \Delta\left(\mathbf{r}_{2}\right) \\
& +\frac{\hbar \beta}{2} \int \prod_{i=1,4} d \mathbf{r}_{i} \Delta^{*}\left(\mathbf{r}_{1}\right) \Delta^{*}\left(\mathbf{r}_{3}\right) B\left(\left\{\mathbf{r}_{i}\right\}\right) \Delta\left(\mathbf{r}_{2}\right) \Delta\left(\mathbf{r}_{4}\right),
\end{aligned}
$$

where $\beta=1 / k_{B} T$ and we have ignored the dependence of $\Delta$ on the imaginary time since we are interested in the classical fluctuations above $T_{c}$. The kernel $A\left(\mathbf{r}_{1}, \mathbf{r}_{2}\right)=$ $g^{-1} \delta\left(\mathbf{r}_{1}-\mathbf{r}_{2}\right)+\hbar^{-1} K\left(\mathbf{r}_{1}, \mathbf{r}_{2}\right)$ is defined through the twoparticle propagator

$$
\begin{aligned}
K\left(\mathbf{r}_{1}, \mathbf{r}_{2}\right) & =-\frac{1}{2} \sum_{i, j} \frac{\tanh \left(\beta \xi_{i} / 2\right)+\tanh \left(\beta \xi_{j} / 2\right)}{\xi_{i}+\xi_{j}} \\
& \times \phi_{i}\left(\mathbf{r}_{1}\right) \phi_{j}\left(\mathbf{r}_{1}\right) \phi_{i}^{*}\left(\mathbf{r}_{2}\right) \phi_{j}^{*}\left(\mathbf{r}_{2}\right)
\end{aligned}
$$

expressed in the Hartree-Fock basis $\left\{\phi_{i}(\mathbf{r})\right\}$ with energies $\left\{\epsilon_{i}\right\}\left(\xi_{i}=\epsilon_{i}-\mu\right)$. The kernel $K$ requires a proper regularization as implied by the use of the contact pseudopotential 13. The kernel of the quartic term is given by

$$
B\left(\left\{\mathbf{r}_{i}\right\}\right)=\hbar \int \prod_{i=1,3} d \tau_{i} G_{0}(1,4) G_{0}(1,2) G_{0}(3,2) G_{0}(3,4) .
$$

where $G_{0}(a, b)$ is the single-particle Green's function and the variable label $a=1, . ., 4$ stands for $\left(\mathbf{r}_{a}, \tau_{a}\right)$.

Pairing fluctuations - In order to evaluate the pair fluctuations $\left\langle|\Delta(\mathbf{r})|^{2}\right\rangle=Z^{-1} \int d\left[\Delta^{*}\right] d[\Delta]|\Delta|^{2} e^{-S\left[\Delta^{*}, \Delta\right] / \hbar}$ it is convenient to decompose $\Delta(\mathbf{r})$ in normal modes

$$
\Delta(\mathbf{r})=\sum_{\nu} \chi_{\nu}(\mathbf{r}) \tilde{\Delta}_{\nu}
$$

where $\chi_{\nu}(\mathbf{r})$ 's are the eigenvectors of the kernel $A[14]$

$$
\int d^{3} r_{1} A\left(\mathbf{r}, \mathbf{r}_{1}\right) \chi_{\nu}\left(\mathbf{r}_{1}\right)=\alpha_{\nu} \chi_{\nu}(\mathbf{r})
$$

For temperatures larger than $T_{c}$ the dominant contribution to the fluctuations comes from the quadratic part of the effective action and in particular, from the lowest eigenvalue $\alpha_{0}$ (and the associated eigenvector $\chi_{0}(\mathbf{r})$ )

$$
\left\langle|\Delta(\mathbf{r})|^{2}\right\rangle \sim \frac{k_{B} T_{c}}{\alpha_{0}}\left|\chi_{0}(\mathbf{r})\right|^{2} .
$$

For a generic system under confinement both $\alpha_{0}$ and $\chi_{0}(\mathbf{r})$ have to be calculated numerically by diagonalization of the kernel $A$.

For an isotropic harmonic trap $V(r)=m \omega^{2} r^{2} / 2$ we proceed now to derive analytical results in the smallsystem limit, i.e. when $R_{T F} \ll \xi$, where $R_{T F}=$ $\left(2 \epsilon_{F} / m \omega^{2}\right)^{1 / 2}$ is the Thomas Fermi radius of the gas and $\xi$ the bulk BCS coherence length estimated at the center of the cloud. Such condition is equivalent to $k_{B} T_{c}^{\text {bulk }} \ll \hbar \omega$, where $T_{c}^{\text {bulk }}$ is the bulk prediction for the critical temperature of the BCS transition evaluated using the central density of the cloud. In this limit the pairing energy is much smaller than the trap level spacing and the Cooper pairs are only formed between particles with quantum numbers $(n, l, m)$ and $(n, l,-m)$ residing in the same harmonic oscillator shell ("intrashell pairing regime") [8]. Since for an isotropic trap the lowest eigenmode is spherically symmetric the eigenvalue equation (6) becomes 15.

$$
\left[1-|g| \alpha_{0}(T)\right] \chi_{0}(r)=\int d r^{\prime} r^{\prime 2} g K\left(r, r^{\prime}\right) \chi_{0}\left(r^{\prime}\right),
$$

where the kernel describing spherically symmetric solutions is

$$
\begin{aligned}
K\left(r, r^{\prime}\right) & =-\frac{1}{8 \pi} \sum_{n, n^{\prime}, l}(2 l+1) \frac{\tanh \left(\beta \xi_{n l}\right)+\tanh \left(\beta \xi_{n^{\prime} l} / 2\right)}{\xi_{n l}+\xi_{n^{\prime} l}} \\
& \times R_{n l}(r) R_{n^{\prime} l}(r) R_{n l}\left(r^{\prime}\right) R_{n^{\prime} l}\left(r^{\prime}\right) .
\end{aligned}
$$

In the small-system limit the effect of the Hartree field is negligible and the $R_{n l}(r)$ 's are approximately the radial eigenfunctions of the isotropic harmonic oscillator. Correspondingly $\xi_{n l} \simeq(n+3 / 2) \hbar \omega-\mu$, where $\mu \simeq \epsilon_{F}=$ $\left(n_{F}+3 / 2\right) \hbar \omega$ is the chemical potential. We further assume that pairing strictly only occurs within the same oscillator shell, and that it mostly occurs about the Fermi surface. In particular, we simplify Eq. (8) by setting $\int d r^{\prime} r^{\prime 2} R_{n l}\left(r^{\prime}\right) R_{n^{\prime} l}\left(r^{\prime}\right) \chi_{0}\left(r^{\prime}\right) \simeq \delta_{n, n^{\prime}} \chi_{n}$ [16], by assuming a weak dependence on $n$ for the relevant shells around the Fermi level, and by taking angular averages. The above assumptions lead to the final expression for the normalized eigenfunction $\chi_{0}(r)$ and the corresponding eigenvalue $\alpha_{0}$ which determine the spatial and temperature dependence of the pairing fluctuations in Eq. (7),

$$
\chi_{0}(r)=\sqrt{\frac{15}{2}} \frac{\left(\pi a_{o s c}\right)^{3 / 2}}{\left(2 n_{F}+3\right)^{5 / 2}} \sum_{l} \frac{(2 l+1)}{4 \pi}\left|R_{n_{F} l}(r)\right|^{2}
$$




$$
\alpha_{0}(T)=\frac{4}{15 \pi^{3}} \frac{\beta_{c} R_{T F}}{a_{o s c}^{4}}\left(1-\frac{T_{c}}{T}\right),
$$

where $T_{c}$ has been computed in Ref. [8], $a_{\text {osc }}=$ $(\hbar / m \omega)^{1 / 2}$ is the harmonic oscillator length and we have kept only the leading temperature dependence in $\alpha_{0}$. An explicit expression for $\chi_{0}(r)$ can be obtained by using that $\sum_{l}(2 l+1)\left|R_{n_{F} l}(r)\right|^{2} / 4 \pi=\partial \rho(r) / \partial n_{F}$, with $\rho(r)$ being the single-component density profile. In the Thomas-Fermi approximation for $\rho(r)$ this yields $\chi_{0}(r)=$ $\left(15 / 8 \pi R_{T F}^{3}\right)^{1 / 2}\left(1-r^{2} / R_{T F}^{2}\right)^{1 / 2}$, showing that the gas is mostly susceptibile to pairing fluctuations in the central part of the trap.

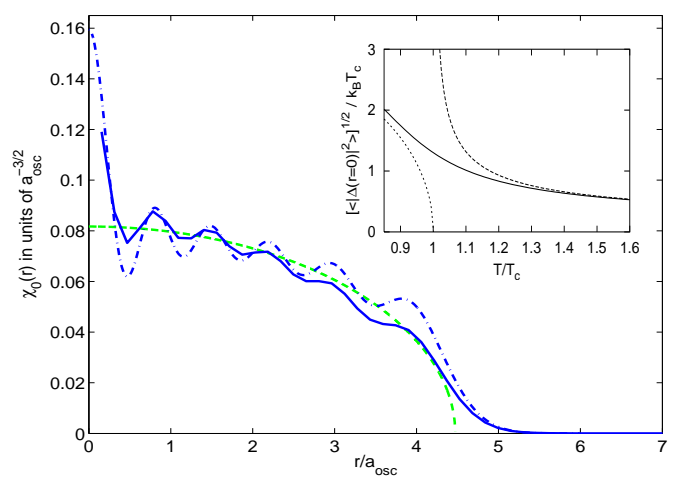

FIG. 1: Lowest eigenvector $\chi_{0}(r)$ (in units of $a_{o s c}^{-3 / 2}$ ) as a function of the radial coordinate $r$ (in units of $a_{o s c}$ ), as obtained from numerical diagonalization (solid line), compared with the analytical solution (10) (dot-dashed line) and with the Thomas-Fermi approximation (dashed line). The inset shows $\left\langle|\Delta(r=0)|^{2}\right\rangle^{1 / 2}$ (in units of $k_{B} T_{c}$ ) as a function of temperature (in units of $T_{c}$ ) as obtained from the quartic (solid line) and the quadratic approximation (dashed line) of the effective action, and the mean-field solution (dotted line).

The spatial behaviour $\chi_{0}(r)$ of the pairing fluctuations is illustrated in Fig. [1 for a system with $n_{F}=$ 10 filled shells ( $\sim 500$ particles) and coupling constant $|g|=0.7 \hbar \omega a_{o s c}^{3}$, as obtained from the analytical expression (10) as well as from the numerical results obtained by diagonalization of the regularized kernel $A$ with a procedure analogous to that described in Ref. 13]. For our choice of parameters $k_{B} T_{c} / \hbar \omega=0.038 \ll 1$, and the system is well within the intrashell regime $\left(T_{c} \simeq 1 \mathrm{nK}\right)$. We find that the analytical expression well approximates the numerical solution, while the characteristic oscillating behavior due to the discrete level structure is lost in the Thomas-Fermi approximation. From the full quantum shell-structure calculations we also predict a parity effect, i.e. that pairing fluctuations should show a maximum or a minimum at $r=0$, depending if $n_{F}$ is even or odd.

At $T=T_{c}$ the lowest eigenvalue $\alpha_{0}$ vanishes, indicating the onset of the superfluid phase. The temperature

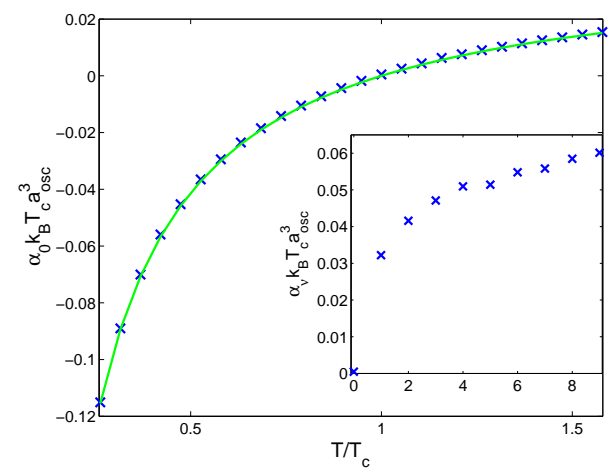

FIG. 2: Lowest eigenvalue $\alpha_{0}(T)$ in units of $\left(k_{B} T_{c}\right)^{-1} a_{\text {osc }}^{-3}$ as a function of temperature (in units of $T_{c}$ ) as calculated numerically ( $\times$ 's) and analytically via Eq. (11) (solid line). The inset shows the lowest eigenvalues $\alpha_{\nu}$ (in the same units as $\alpha_{0}$ ) as functions of the index $\nu$, evaluated at $T \sim T_{c}$.

behaviour of $\alpha_{0}$ is illustrated in Fig. 22 where we compare the approximate expression (11) with the exact numerical solutions for the same choice of parameters as in Fig. [1] The figure shows an excellent agreement between the two calculations.

Since $\alpha_{0}\left(T_{c}\right)=0$, according to Eq. (77) the pairing fluctuations diverge at $T_{c}$ (dashed line in the inset of Fig. (1). This is an artifact due to the quadratic approximation used for the effective action, and can be cured by taking into account the quartic term [10], which we estimate from Eq. (2) within a single-mode approximation. This is well justified by noticing that the lowest eigenvalue is significantly smaller than the other ones (inset in Fig. 22), and thus the $\nu=0$ mode strongly dominates the fluctuations in the intrashell regime. The effective action then reduces to the simple form

$$
S_{\mathrm{eff}} \simeq \hbar \beta_{c}\left[\alpha_{0}\left|\tilde{\Delta}_{\nu=0}\right|^{2}+\frac{1}{2} B_{0}\left(T_{c}\right)\left|\tilde{\Delta}_{\nu=0}\right|^{4}\right] .
$$

where $B_{0}\left(T_{c}\right) \simeq 64 \beta_{c}^{3} / 675 \pi^{6} n_{F} a_{\text {osc }}^{6}$ and we have only retained the contribution to the Green's functions in Eq. (4) coming from the levels at the Fermi surface, consistently with the intrashell pairing approximations. By introducing the dimensionless quantity $\Delta_{\nu=0} /\left(a_{\text {osc }}^{3 / 2} k_{B} T_{c}\right), S_{\text {eff }}$ only depends on $n_{F}$ and $T / T_{c}$, since $R_{T F} / a_{o s c}=\left(2 n_{F}+3\right)^{1 / 2}$. The value of $n_{F}$ controls the magnitude of the critical region around $T_{c}$, which we estimate to be $\delta T / T_{c} \sim 2 /\left(n_{F}+1\right)\left(n_{F}+2\right)$ from the Ginzburg criterion.

Using the effective action Eq. (12) the temperature behaviour of the pairing fluctuations can be estimated analytically, and is illustrated in the inset of Fig. 10 (solid line). The figure shows that the divergence predicted by the quadratic term alone is indeed eliminated and the behavior is now smooth across $T_{c}$, tending to the meanfield result (dotted line) below $T_{c}$. We should remark that Eq. (12) is only accurate close enough to $T_{c}$, while 
well below $T_{c}$ the full action should be withheld in the averaging.

We also mention that in the large-system limit $\xi \ll$ $R_{T F}$ pairing fluctuations can be described along the lines of Ref. [17]. We do not pursue the issue further in this work since in this limit they are expected to be small.

Density correlations - The presence of Cooper pairs implies non-zero density-density correlations among particles with opposite spins located at $\mathbf{q},-\mathbf{q}$. Altman et al. 11] suggested that such "anomalous" correlations could be measured probing the density-density correlation function after the trap has been released and the gas has undergone a ballistic expansion: $\left\langle n_{\sigma}(\mathbf{r}) n_{-\sigma}\left(\mathbf{r}^{\prime}\right)\right\rangle \sim$ $\left\langle n_{\sigma}\left(\mathbf{q}_{\mathbf{r}}\right) n_{-\sigma}\left(\mathbf{q}_{\mathbf{r}^{\prime}}\right)\right\rangle$, where $\mathbf{q}_{\mathbf{r}}=(m / \hbar t) \mathbf{r}, m$ is the mass of the fermions and $t$ is the time lapse after the trap has been opened. At mean-field level it can be shown that $\left\langle\delta n_{\sigma}(\mathbf{r}) \delta n_{-\sigma}(-\mathbf{r})\right\rangle \propto\left|\Delta_{B C S}\right|^{2}$, with $\delta n_{\sigma}(\mathbf{r})=n_{\sigma}(\mathbf{r})-$ $\left\langle n_{\sigma}(\mathbf{r})\right\rangle$, and hence, one could directly access the gap field amplitude. Above $T_{c}$ the same density-density correlation function is also sensitive to pairing fluctuations. Proceeding as for obtaining Eq. (2) for a uniform system we get $\left\langle\delta n_{\sigma}(\mathbf{r}) \delta n_{-\sigma}(-\mathbf{r})\right\rangle=\left\langle\left\langle\left(\Delta / 2 E_{q_{r}}\right)^{2}[1-\right.\right.$ $\left.\left.\left.2 f\left(E_{q_{r}}\right)\right]^{2}\right\rangle\right\rangle_{S_{e f f}}$, where $\langle\langle\cdot\rangle\rangle_{S_{\text {eff }}}$ means the average over the distribution of $\Delta$ weighted by the effective action $S_{\text {eff }}, f(x)=\{\exp [\beta(x-\mu)]+1\}^{-1}$ is the Fermi distribution and $E_{q}=\left(\hbar^{4} q^{4} / 4 m^{2}+\Delta^{2}\right)^{1 / 2}$ is the usual BCS quasi-particle dispersion relation.

In small harmonically trapped systems anomalous correlations occur between particles with opposite spins and quantum numbers $(n, l, m)$ and $(n, l,-m)$. In the intrashell limit analogous approximations to those used in order to obtain Eqs. (10) and (11) yield

$$
\left\langle\delta n_{\sigma}(\mathbf{r}) \delta n_{-\sigma}(-\mathbf{r})\right\rangle=\frac{1}{4}\left(\frac{\partial \rho\left(q_{r}\right)}{\partial n_{F}}\right)^{2}\left\langle\left\langle[F(\Delta)]^{2}\right\rangle\right\rangle_{S_{e f f}}
$$

where $F(\Delta)=1-2 f(|\Delta|)+2(|\Delta| / \hbar \omega) \log \left(\gamma n_{F}\right)$, with $\gamma=e^{C} \simeq 1.78$, and $C$ Euler's constant. In this limit the spatial dependence of the anomalous correlations is proportional to $\left|\chi_{0}(r)\right|^{2}$, which is given by Eq. (10) and shown in Fig. 1] Also for the small system the meanfield result is non-zero only below $T_{c}$, where we find $\left\langle\delta n_{\sigma}(\mathbf{r}) \delta n_{-\sigma}(-\mathbf{r})\right\rangle \propto\left|\Delta_{B C S}(\mathbf{r})\right|^{2}$.

The effect of pairing fluctuations is not confined to the density-density correlations described in this work. As another example, we expect a soft gap in the laser induced tunneling (discussed in Ref. [18]) above the critical temperature.

The authors would like to thank Prof. M. Tosi for helpful discussions. This work has been supported by the EU (RTN-Nanoscale). LV wishes to thank the SNS, where part of this work was carried out, for the kind hospitality.
AM wishes to thank the LPTMS Orsay, where this work was completed, for the kind hospitality.

[1] B. DeMarco, S.B. Papp, and D.S. Jin, Phys. Rev. Lett. 86, 5409 (2001); A.G. Truscott et al., Science 291, 2570 (2001); F. Schreck et al., Phys. Rev. A 64, 011402 (2001); S.R. Granade et al., Phys. Rev. Lett. 88, 120405 (2002); Z. Hadzibabic et al., ibid. 88, 160401 (2002).

[2] P. Nozières and S. Schmitt-Rink, J. Low. Temp. Phys. 59, 195 (1985); C.A. R. Sá de Melo et al., Phys. Rev. Lett. 713202 (1993); R. Combescot, ibid. 83, 3766 (1999); E. Timmermans, et al., Phys. Lett. A 285, 228 (2001); M. Holland et al., Phys. Rev. Lett. 87, 120406 (2001); Y. Ohashi and A. Griffin, ibid. 89, 130402 (2002), J. Carlson et al., ibid. 91, 050401 (2003).

[3] K. M. O'Hara et al., Science 298, 2179 (2002); C. A. Regal and D. S. Jin, Phys. Rev. Lett. 90, 230404 (2003); T. Bourdel et al., ibid. 91, 020402 (2003); C. A. Regal et al., Nature 404, 47 (2003); J. Cubizolles et al., Phys. Rev. Lett.91, 240401 (2003).

[4] M. Greiner, C. A. Regal, D. S. Jin, cond-mat/0311172 S. Jochim, et al., Science 302, 2101 (2003), M. W. Zwierlein et al., Phys. Rev. Lett. 91250401 (2003);

[5] C. A. Regal et al. Phys. Rev. Lett. 92, 040403 (2004); M. Bartenstein, et al., cond-mat/0401109

[6] L. D. Carr, G. V. Shlyapnikov and Y. Castin, cond-mat/0308306

[7] H.T.C. Stoof et al., Phys. Rev. Lett. 76, 10 (1996); H. Heiselberg et al., ibid. 85, 2418 (2000); H. Heiselberg and B. Mottelson, ibid. 88, 190401 (2002).

[8] G. M. Bruun and H. Heiselberg, Phys. Rev. A 65, 053407 (2002); G. M. Bruun, ibid. 66, 041602 (2002).

[9] A.I. Larkin and A.A.Varlamov, in Handbook on Superconductivity: Conventional and Unconventional Superconductors, edited by K.-H.Bennemann and J.B. Ketterson, Springer, 2002.

[10] B. Mühlschlegel, D.J. Scalapino and R. Denton, Phys. Rev. B 6, 1767 (1972).

[11] E. Altman, E. Demler, and M.D. Lukin, cond-mat/0306226

[12] H. Kleinert, Forts. Phys. 26, 565 (1978).

[13] G. M. Bruun, et al., Eur. Phys. J. D 7, 433 (1999), Eq. (28).

[14] Since the operator $\int d^{3} r_{1} A_{r e g}\left(\mathbf{r}, \mathbf{r}_{1}\right) \cdot$ is self-adjoint, the eigenvectors corresponding to different $\alpha_{\nu}$ 's are orthogonal. We choose the normalization $\int d^{3} r\left|\chi_{\nu}(\mathbf{r})\right|^{2}=1$.

[15] In the derivation of the analytical results in place of the regularization of the kernel we use a sum cutoff. Such procedure is justified a posteriori by comparison with the numerical results based on the proper renormalization scheme.

[16] This follows from the oscillating nature of $R_{n l}(r)$ combined with the fact that $\chi_{0}(r)$ has a definite sign.

[17] M. A. Baranov and D. S. Petrov, Phys. Rev. A 58, R801 (1998).

[18] P. Torma and P. Zoller, Phys. Rev. Lett 85, 487 (2000). 